INPLASY

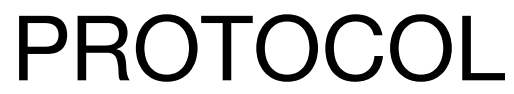

To cite: Wei et al. Different acupuncture therapies combined with rehabilitation in the treatment of

scapulohumeral periarthritis:

A protocol for systematic review and network metaanalysis. Inplasy protocol 202090035. doi:

10.37766/inplasy2020.9.0035

Received: 08 September 2020

Published: 08 September 2020

Corresponding author:

Xinju Hou

2289527835@qq.com

Author Affiliation:

Nanchang Hongdu Hospital of

Traditional Chinese Medicine

Support: None.

Review Stage at time of this submission: Preliminary searches.

Conflicts of interest: None.

\section{Different acupuncture therapies combined with rehabilitation in the treatment of scapulohumeral periarthritis: A protocol for systematic review and network meta-analysis}

Wei, L1; Zhu, M2; Peng, T3; Xiong, W4; Hou, X5.

Review question / Objective: Whether different acupuncture and moxibustion combined with rehabilitation in the treatment of scapulohumeral periarthritis is better than simple rehabilitation?

Condition being studied: Scapulohumeral periarthritis, commonly known as frozen shoulder, is a common and disabling musculoskeletal disease in middle-aged people. It usually refers to shoulder pain syndrome caused by soft tissue injury around the shoulder joint and the disease with limited motor function.It is known as "Fifty shoulders" and "shoulder congealing syndrome" in traditional Chinese medicine. Its main clinical manifestation is shoulder pain. At present, the main non-surgical treatment of scapulohumeral periarthritis is oral non steroidal drugs and intra-articular injection, but most of them disappear within 4 weeks after treatment. In recent years, the systematic evaluation of acupuncture intervention on scapulohumeral periarthritis is increasing gradually, and the curative effect is better. At the same time, various kinds of acupuncture and moxibustion are involved. However, for clinicians, whether acupuncture can be used as a supplementary alternative therapy for scapulohumeral periarthritis and what kind of acupuncture therapy should be selected in clinical practice has no evidence-based basis.

INPLASY registration number: This protocol was registered with the International Platform of Registered Systematic Review and Meta-Analysis Protocols (INPLASY) on 08 September 2020 and was last updated on 08 September 2020 (registration number INPLASY202090035).

\section{INTRODUCTION}

Review question / Objective: Whether different acupuncture and moxibustion combined with rehabilitation in the treatment of scapulohumeral periarthritis is better than simple rehabilitation? 
Condition being studied: Scapulohumeral periarthritis, commonly known as frozen shoulder, is a common and disabling musculoskeletal disease in middle-aged people. It usually refers to shoulder pain syndrome caused by soft tissue injury around the shoulder joint and the disease with limited motor function.It is known as "Fifty shoulders" and "shoulder congealing syndrome" in traditional Chinese medicine. Its main clinical manifestation is shoulder pain. At present, the main non-surgical treatment of scapulohumeral periarthritis is oral non steroidal drugs and intra-articular injection, but most of them disappear within 4 weeks after treatment. In recent years, the systematic evaluation of acupuncture intervention on scapulohumeral periarthritis is increasing gradually, and the curative effect is better. At the same time, various kinds of acupuncture and moxibustion are involved. However, for clinicians, whether acupuncture can be used as a supplementary alternative therapy for scapulohumeral periarthritis and what kind of acupuncture therapy should be selected in clinical practice has no evidence-based basis.

\section{METHODS}

Participant or population: The patients diagnosed with scapulohumeral periarthritis meet the diagnostic criteria of "diagnostic efficacy standard of traditional Chinese medicine", with clear curative effect standard, without limitation of age, race, gender and case source.

Intervention: The experimental group was treated with electroacupuncture, fire acupuncture, warm acupuncture or floating acupuncture.

Comparator: The control group was treated with simple rehabilitation therapy.

Study designs to be included: Randomized controlled trial.

Eligibility criteria: Literature exclusion criteria: (1) non RCTs literature, such as case reports, literature review, etc.; 2) acupuncture therapy except for 4 kinds of acupuncture; (3) other interference therapies in the experimental group and the control group; (4) only one article with the most complete information was selected for the repeatedly detected and repeatedly published literature; (5) the literature with incomplete data or unable to obtain data and full text; (6) the literature suspected of falsification.

Information sources: Chinese databases including CNKI, Wan-Fang, VIP and CBM were searched. PubMed and Cochrane library were searched in English.The retrieval time is from the establishment of the database to September 8, 2020. All of the retrieval strategies are in the form of subject words + free words, and relevant references are retrieved manually. The English key words are "moxa warming needling treatment", "fire need acupunture", "electroacupuncture", "floating need", "periarthritis of shoulder", "scapulohumeral periathritis", "frozen shoulder", "50 shoulder".

Main outcome(s): The included outcome indicators included one or more of the following: cure rate, total effective rate, visual analogue scale (VAS) score, shoulder function score.

Data management: First, two reviewers independently screened according to the inclusion and exclusion criteria of the literature, and then cross checked. In case of disagreement, the third evaluator could make a decision. The extracted information includes: author, publication time, number of cases, allocation method, intervention measures, course of treatment and outcome indicators.

Quality assessment / Risk of bias analysis: Two reviewers independently assessed the bias risk of the final included articles according to the Cochrane reviewer bias risk assessment tool, including selection bias, implementation bias, measurement bias, follow-up bias, reporting bias and other sources bias. The evaluation results were "high risk" and "low risk" Risk) and "unclear risk". 
Strategy of data synthesis: Revman $\mathbf{5 . 3}$ software was used for bias evaluation and direct meta-analysis. The outcome indicators were count data, odds ratio (or), mean difference (MD) for measurement data, and $95 \%$ confidence interval $(95 \% \mathrm{CI})$ for effect. For heterogeneity test, if $12<$ $50 \%, P>0.10$, it indicates that there is no obvious heterogeneity, and the fixed effect model is selected to merge the effect; if the combined data is $12>50 \%, P<0.10$, it indicates that the heterogeneity is high, and the random effect model is selected to merge the effect quantity. Gemtc 0.14.3 and stata14.2 are used for mesh meta analysis. In gemtc software, Markov chain Monte Carlo (MCMC) method is used for Bayesian mesh meta analysis. Four chains are used for simulation. The number of iterations is set to 50000, and the first 20000 annealing times are used to eliminate the influence of initial value, and the step size is set to 10 . At the same time, potential scale reduced factor (psrf) is used to evaluate the convergence of the results. When the PRSF is close to or equal to 1.00 (1.00 $\leq$ PRSF $\leq$ $1,05)$, it shows that the convergence of the results is good and the reliability of the results is high. If the PRSF is not in this range, the number of iterations will be increased by 50000 times manually until FRSF is within this range.

Subgroup analysis: If the $12>50 \%$, we will explore sources of heterogeneity by subgroup analysis and meta-regression, the STATA software will be used for subgroup analysis and meta-regression analysis according to the characteristics of the test.

Sensibility analysis: Sensitivity analysis will be done by changing the effect model or deleting one study at a time to investigate the impact of individual studies on the overall set. We will evaluate the robustness of meta-analysis results through sensitivity analysis, and exclude small sample trials and low-quality trials, and explore the impact of trial quality on efficacy estimation.
Keywords: scapulohumeral periarthritis; acupuncture; rehabilitation; network meta analysis; protocol.

Contributions of each author:

Author 1 - Lingzhi Wei.

Author 2 - Manhua Zhu.

Author 3 - Tianzhong Peng.

Author 4 - Wei Xiong.

Author 5 - Xinju Hou.

Country(ies) involved: China. 